\title{
Implement of multiple access technique by wireless power transfer and relaying network
}

\author{
Anh-Tu Le ${ }^{1}$, Dinh-Thuan Do ${ }^{2}$ \\ ${ }^{1}$ Faculty of Electronics Technology, Industrial University of Ho Chi Minh City, Vietnam \\ ${ }^{2}$ Wireless Communications Research Group, Faculty of Electrical \& Electronics Engineering, Ton Duc Thang University, \\ Ho Chi Minh City, Vietnam
}

\begin{tabular}{|c|c|}
\hline Article Info & ABSTRACT \\
\hline Article history: & \multirow{8}{*}{$\begin{array}{l}\text { In this paper, we investigate non-orthogonal multiple access (NOMA) network relying } \\
\text { on wireless power transfer to prolong lifetime. The base station (BS) sends common } \\
\text { signals to the relay with two functions (energy harvesting (EH) and signal processing) } \\
\text { to further serve two NOMA users in downlink. Performance gap exists since different } \\
\text { power allocation factor assigned from power splitting protocol adopted at the relay and } \\
\text { such relay employs both amplify-and-forward (AF) and decode-and-forward schemes. } \\
\text { To provide performance metrics, we prove formulas of the outage probability which } \\
\text { is a function of transmit signal to noise ratio. Simulation results indicate specific } \\
\text { parameters to adjust system performance of two user in the considered EH-NOMA } \\
\text { system. This finding is important recommendation to design EH-NOMA which shows } \\
\text { particular outage performance at required target rates. }\end{array}$} \\
\hline Received Oct 16, 2020 & \\
\hline Revised Jan 7, 2021 & \\
\hline Accepted Feb 16, 2021 & \\
\hline Keywords: & \\
\hline NOMA & \\
\hline Outage $\mathrm{p}$ & \\
\hline & \\
\hline
\end{tabular}

This is an open access article under the CC BY-SA license.

Corresponding Author:

Dinh-Thuan Do

Wireless Communications Research Group

Faculty of Electrical \& Electronics Engineering, Ton Duc Thang University

Ho Chi Minh City, Vietnam

Email: dodinhthuan@tdtu.edu.vn

\section{INTRODUCTION}

Recently, non-orthogonal multiple access (NOMA) has proposed as a leading multiple access technique to implement various networks in the fifth generation $(5 \mathrm{G})$ wireless communication. NOMA provides lots of improvements such as massive connections, higher spectral efficiency, guaranteed user fairness, and low access latency [1], [2]. In principle, NOMA relying on non-orthogonal resource to serve many users in same time and this scheme is different with conventional orthogonal multiple access (OMA) relaying networks [3]-[6]. Popular schemes are employed in NOMA such as frequency-division multiple access (FDMA) and time-division multiple access (TDMA). The challenging models are developed by the authors in [7]-[9], in which combination between relay schemes and NOMA is introduced as novel scheme, namely cooperative NOMA. The novel idea of NOMA is to devide the power domain for realizing MA. In NOMA, different power levels are allocated for different users with respect to distinguished signals [10]-[12]. To further detect signal from received mixture signal, the successive interference cancellation (SIC) is required to eliminate the multiuser interference at the receivers [13]. To further increase spectrum efficiency, cognitive radio (CR) can be integrated with NOMA [14].

The authors in [15]-[21] investigated important role of EH in deployment of NOMA.In the context of EH-NOMA, the secondary users receive the radio-frequency signals to harvest energy and to securely transmit 
the secondary privacy information with advantage of the NOMA approach [15]. Different from the traditional ideal linear EH scheme, the authors in [15] implemented the practical non-linear EH architecture. The authors in [16] studied a multiple-access channel (MAC), in which transmitters with capability of EH. The batteries equipped in the transmitters have non-ideal charging and discharging characteristics, which leads to a fractional loss of power driven [16]. Two modes of CR-NOMA are studied in [16] including underlay network and overlay network. Such NOMA system relying on Simultaneous Wireless Information and Power Transfer (SWIPT) architecture to form a SWIPT-aided CR-NOMA system model which exhibits better performance with the approximate formula of energy efficiency for two modes are derived.

Motivated by recent works [2], [7], [8], this paper considers a new cooperative EH-NOMA protocol, where the intermediate relay has not equipped the fixed power source and acts as a wireless powered relay with respect to optimal instantaneous rate. Such relay is designed to help signal forwarding to representative weak user and strong user in NOMA.

\section{SYSTEM MODEL AND SNR COMPUTATION}

\subsection{System model}

The considered system model in Figure 1 comprises of a base station (BS), two destinations $\left(U_{1}, U_{2}\right)$ and a power splitting-based EH relay [18]-[21]. The channel gains $h_{a b}$ for link from node $a$ to node $b$ corresponding exponential distribution with means $\lambda_{a b}$. It is assumed that the channels are quasistatic which means that the channels remains constant over one transmission time while different values for these channels over different transmission times. The Amplify-and-Forward (AF) or Decode-and-Forward (DF) relaying protocol is employed at relay. $T$ is called as the whole transmission time and it is divided into two transmission phases. Regarding EH function, it uses only the harvested power during the first transmission phase to transmit during the second transmission phase. $P_{S}$ is the transmit power of the BS, $x_{1}$ and $x_{2}$ are the messages BS intends to send to the weak user $U_{1}$ and the strong user $U_{2} . a_{1}$ and $a_{2}$ are denoted as the power allocation coefficients in NOMA scheme. Following the principle of NOMA, we assume that $a_{1}>a_{2}$ with $a_{1}+a_{2}=1$.
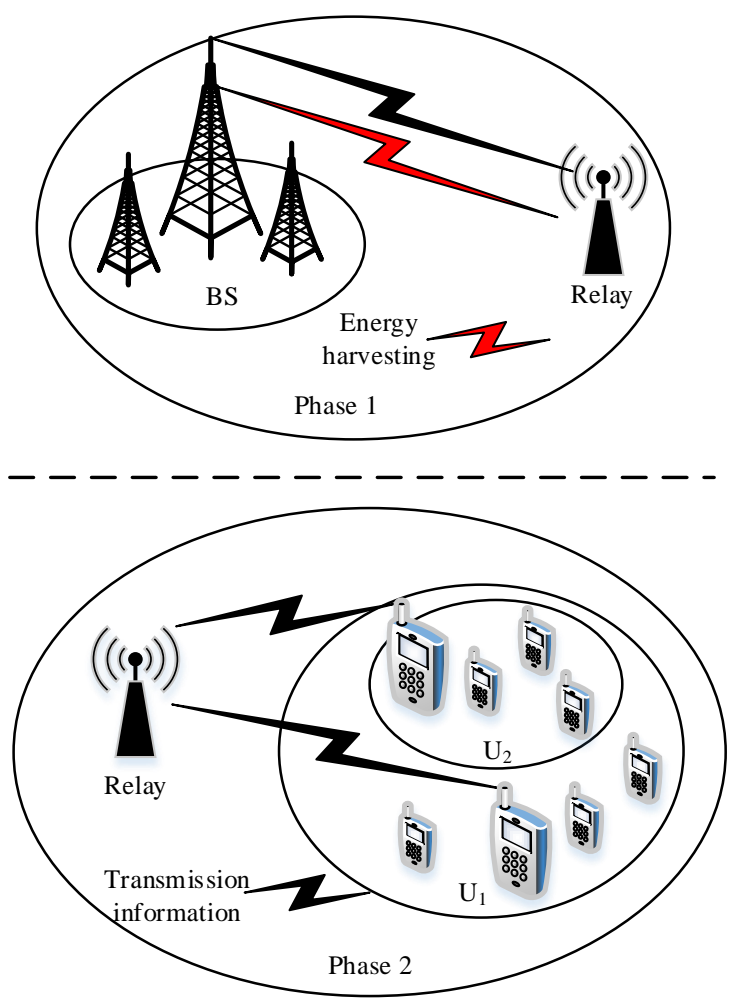

Figure 1. System model of wireless powered NOMA 


\subsection{SNR computation}

In the first transmission phase, the received signal at the relay $R$ is expressed by

$$
y_{R}=\sqrt{(1-\beta)} h_{S R}\left(\sqrt{a_{1} P_{S}} x_{1}+\sqrt{a_{2} P_{S}} x_{2}\right)+n_{R}
$$

where $n_{R}$ stands for the additive white Gaussian noise (AWGN) with variance $N_{0}$, PS coefficient is denoted by $\beta(0<\beta<1)$ assigned at relay.

The harvested power during the first transmission phase is given as [20, 25]

$$
P_{h}=\beta P_{S}\left|h_{S R}\right|^{2}
$$

Thus, the signal-to-interference-plus-noise ratio (SINR) and signal-to-noise ratio (SNR) after performing successive interference cancellation (SIC) in NOMA can be determined to detect signal $x_{1}, x_{2}$ respectively at $\mathrm{R}$ and they can be given as

$$
\begin{aligned}
\gamma_{S R 1} & =\frac{(1-\beta) a_{1} P_{S}\left|h_{S R}\right|^{2}}{(1-\beta) a_{2} P_{S}\left|h_{S R}\right|^{2}+N_{0}} \\
& =\frac{(1-\beta) a_{1} \rho\left|h_{S R}\right|^{2}}{(1-\beta) a_{2} \rho\left|h_{S R}\right|^{2}+1},
\end{aligned}
$$

and

$$
\gamma_{S R 2}=(1-\beta) a_{2} \rho\left|h_{S R}\right|^{2},
$$

where $\rho=\frac{P_{S}}{N_{0}}$ is so-called as transmit SNR at the BS.

Then, the SINR to detect $x_{1}$ at user $U_{1}$ is given by

$$
\gamma_{R U_{1}}=\frac{\beta \rho a_{1}\left|h_{R U_{1}}\right|^{2}\left|h_{S R}\right|^{2}}{\beta \rho a_{2}\left|h_{S R}\right|^{2}\left|h_{R U_{1}}\right|+1}
$$

Similarly, the SINR to detect $x_{2}$ at user $U_{2}$ is computed by

$$
\gamma_{R U_{2,1}}=\frac{\beta \rho a_{1}\left|h_{R U_{2}}\right|^{2}\left|h_{S R}\right|^{2}}{\beta \rho a_{2}\left|h_{S R}\right|^{2}\left|h_{R U_{2}}\right|+1} .
$$

After SIC implementation at $U_{2}$, SNR to to detect $x_{2}$ is

$$
\gamma_{R U_{2}}=\beta \rho a_{2}\left|h_{R U_{2}}\right|^{2}\left|h_{S R}\right|^{2} .
$$

\subsection{Mode of decode-and-forward relaying}

With regard to EH-NOMA using DF relaying the end-to-end SNR from the BS to user $U_{1}$ can be expressed as

$$
\begin{aligned}
\gamma_{U_{1}}^{D F} & =\min \left(\gamma_{S R 1}, \gamma_{R U_{1}}\right) \\
& =\min \left(\frac{(1-\beta) a_{1} \rho\left|h_{S R}\right|^{2}}{(1-\beta) a_{2} \rho\left|h_{S R}\right|^{2}+1}, \frac{\beta \rho a_{1}\left|h_{R U_{1}}\right|^{2}\left|h_{S R}\right|^{2}}{\beta \rho a_{2}\left|h_{S R}\right|^{2}\left|h_{R U_{1}}\right|+1}\right)
\end{aligned}
$$

It is worth noting that such $\min$ function is maximized when all of its argument becomes equal. As a result, the following optimal value of $\beta$ is found to achieve maximal instantaneous rate as

$$
\beta_{D F, U_{1}}^{*}=\frac{1}{\left|h_{R U_{1}}\right|^{2}+1}
$$

For DF case, the SNR from the BS to $U_{2}$ can be computed as

$$
\begin{aligned}
\gamma_{U_{2}}^{D F} & =\min \left(\gamma_{S R 2}, \gamma_{R U_{2}}\right) \\
& =\min \left((1-\beta) a_{2} \rho\left|h_{S R}\right|^{2}, \beta \rho a_{2}\left|h_{R U_{2}}\right|^{2}\left|h_{S R}\right|^{2}\right) .
\end{aligned}
$$

Similarly, the optimal value of $\beta$ can be obtained to achieve optimal instantaneous rate as

$$
\beta_{D F, U_{2}}^{*}=\frac{1}{\left|h_{R U_{2}}\right|^{2}+1} .
$$

Implement of multiple access technique by wireless power transfer and relaying network (Anh-Tu Le) 


\subsection{Mode of amplify-and-forward relaying}

For AF- based EH-NOMA, the received SNR at the $U_{1}$ can be formulated by (4) [22]

$$
\begin{aligned}
\gamma_{U_{1}}^{A F} & \simeq \frac{\gamma_{S R 1} \gamma_{R U_{1}}}{\gamma_{S R 1}+\gamma_{R U_{1}}} \\
& \simeq \frac{(1-\beta) \beta \rho a_{1}\left|h_{R U_{1}}\right|^{2}\left|h_{S R}\right|^{2}}{2(1-\beta) \beta \rho a_{2}\left|h_{S R}\right|^{2}\left|h_{R U_{1}}\right|+(1-\beta)+\beta\left|h_{R U_{1}}\right|^{2}}
\end{aligned}
$$

Similarly, the SINR can be obtained at $U_{2}$ as

$$
\gamma_{U_{2}}^{A F} \simeq \frac{(1-\beta) \beta \rho a_{2}\left|h_{R U_{2}}\right|^{2}\left|h_{S R}\right|^{2}}{(1-\beta)+\beta\left|h_{R U_{2}}\right|^{2}}
$$

The following optimal value of $\beta$ in AF scenario is expressed by

$$
\beta_{A F, U_{i}}^{*}=\frac{1}{\left|h_{R U_{i}}\right|+1}
$$

\section{OUTAGE PROBABILITY}

\subsection{Decode-and-forward relaying}

\subsubsection{Outage probability of $U_{1}$}

As definition and considering as important metric reported in [7], [8], this section presents outage performance of such EH-NOMA.

Putting optimal value of $\beta$ raised in (9) into (8), it can be obtained SINR in new form as

$$
\gamma_{U_{1}}^{D F}=\frac{\rho a_{1}\left|h_{S R}\right|^{2}\left|h_{R U_{1}}\right|^{2}}{\rho a_{2}\left|h_{S R}\right|^{2}\left|h_{R U_{1}}\right|^{2}+\left|h_{R U_{1}}\right|^{2}+1}
$$

Then, the outage probability of $U_{1}$ can be formulated as

$$
O P_{U_{i}}^{D F}=\operatorname{Pr}\left(\gamma_{U_{i}}^{D F}<\gamma_{t h i}\right)
$$

where $\gamma_{t h i}=2^{2 R_{i}}-1, i \in\{1,2\}$, with $R_{i}$ is target rates for $U_{1}, U_{2}$, respectively.

Proposition 1: The expected outage of $U_{1}$ is given by

$$
\begin{aligned}
O P_{U_{1}}^{D F} & =\int_{0}^{\infty}\left(1-e^{-\frac{\gamma_{t h 1}(x+1)}{\left(a_{1}-\gamma_{t h 1} a_{2}\right) \rho x}}\right) \lambda_{R U_{1}} e^{-\lambda_{R U_{1}} x} d x \\
& =1-2 e^{-\frac{\lambda_{S R} \gamma_{t h 1}}{\left(a_{1}-\gamma_{t h 1} a_{2}\right) \rho}} \sqrt{\frac{\gamma_{t h 1} \lambda_{S R} \lambda_{R U_{1}}}{\left(a_{1}-\gamma_{t h 1} a_{2}\right) \rho}} K_{1}\left(2 \sqrt{\frac{\gamma_{t h 1} \lambda_{R U_{1}} \lambda_{S R}}{\left(a_{1}-\gamma_{t h 1} a_{2}\right) \rho}}\right)
\end{aligned}
$$

Proof: See appendix A

\subsubsection{Outage probability of $U_{2}$}

Similarly, originating from SINR we have

$$
\gamma_{U_{2}}^{D F}=\frac{\rho a_{2}\left|h_{R U_{2}}\right|^{2}\left|h_{S R}\right|^{2}}{\left|h_{R U_{2}}\right|^{2}+1}
$$

Then, after some algebra mathematical manipulations, the outage probability of $U_{2}$ can be computed as

$$
\begin{aligned}
O P_{U_{2}}^{D F} & =1-2 e^{-\frac{\lambda_{S R} \gamma_{t h 2}}{\rho a_{2}}} \sqrt{\frac{\lambda_{S R} \lambda_{R U_{2}} \gamma_{t h 2}}{\rho a_{2}}} \\
& \times K_{1}\left(2 \sqrt{\frac{\lambda_{S R} \lambda_{R U_{2}} \gamma_{t h 2}}{\rho a_{2}}}\right)
\end{aligned}
$$

where $K_{n}($.$) stands for the modified Bessel function of second kind and n$th order. 


\subsection{Amplify-and-Forward relaying}

\subsubsection{Outage probability of $U_{1}$}

After putting the optimal PS ratio $\beta$ of AF based EH-NOMA we can write the SINR for this case and it is expressed in approximate form as

$$
\gamma_{U_{1}}^{A F} \simeq \frac{\rho a_{1}\left|h_{R U_{1}}\right|^{2}\left|h_{S R}\right|^{2}}{2 \rho a_{2}\left|h_{S R}\right|^{2}\left|h_{R U_{1}}\right|^{2}+\left(1+\left|h_{R U_{1}}\right|\right)^{2}}
$$

Then, the expected outage performance at $U_{1}$ can be formulated by

$$
\begin{aligned}
O P_{U_{1}}^{A F} & \simeq \operatorname{Pr}\left(\gamma_{U_{1}}^{A F}<\gamma_{t h 1}\right) \\
& \simeq \operatorname{Pr}\left(\left|h_{S R}\right|^{2}<\frac{\gamma_{t h 1}\left(1+\left|h_{R U_{1}}\right|\right)^{2}}{\rho\left(a_{1}-2 \gamma_{t h 1} a_{2}\right)\left|h_{R U_{1}}\right|^{2}}\right)
\end{aligned}
$$

Performing computational technique reported in [7], it can be obtained outage probability for $U_{1}$ in AF mode as

$$
\begin{aligned}
O P_{U_{1}}^{A F} & \simeq 1-2 e^{-\frac{\lambda_{S R} \gamma_{t h 1}}{\rho\left(a_{1}-2 \gamma_{t h 1} a_{2}\right)}} \sqrt{\frac{\gamma_{t h 1} \lambda_{S R} \lambda_{R U_{1}}}{\rho\left(a_{1}-2 \gamma_{t h 1} a_{2}\right)}} \\
& \times K_{1}\left(2 \sqrt{\frac{\gamma_{t h 1} \lambda_{S R} \lambda_{R U_{1}}}{\rho\left(a_{1}-2 \gamma_{t h 1} a_{2}\right)}}\right)+e^{-\frac{\lambda_{S R} \gamma_{t h 1}}{\rho\left(a_{1}-2 \gamma_{t h 1} a_{2}\right)}} \\
& \times \int_{0}^{\infty} \frac{2 \gamma_{t h 1} \lambda_{S R}}{\rho\left(a_{1}-2 \gamma_{t h 1} a_{2}\right) \sqrt{x}} e^{-\frac{-\lambda_{S R} \gamma_{t h 1}}{\rho\left(a_{1}-2 \gamma_{t h 1} a_{2}\right) x}} e^{-\lambda_{R U_{1}} x} d x
\end{aligned}
$$

With help [23, 3.471.9], (22) can be formulated as

$$
\begin{aligned}
O P_{U_{1}}^{A F} & \simeq 1-2 e^{-\frac{\lambda_{S R} \gamma_{t h 1}}{\rho\left(a_{1}-2 \gamma_{t h 1} a_{2}\right)}} \sqrt{\frac{\gamma_{t h 1} \lambda_{S R} \lambda_{R U_{1}}}{\rho\left(a_{1}-2 \gamma_{t h 1} a_{2}\right)}} \\
& \times K_{1}\left(2 \sqrt{\frac{\gamma_{t h 1} \lambda_{S R} \lambda_{R U_{1}}}{\rho\left(a_{1}-2 \gamma_{t h 1} a_{2}\right)}}\right)+e^{-\frac{\lambda_{S R} \gamma_{t h 1}}{\rho\left(a_{1}-2 \gamma_{t h 1} a_{2}\right)}} \frac{4 \gamma_{t h 1} \lambda_{S R} \lambda_{R U_{1}}^{2}}{\rho\left(a_{1}-2 \gamma_{t h 1} a_{2}\right)} \\
& \times\left(\frac{\gamma_{t h 1} \lambda_{S R}}{\rho\left(a_{1}-2 \gamma_{t h 1} a_{2}\right) \lambda_{R U_{1}}}\right)^{1 / 4} K_{1 / 2}\left(2 \sqrt{\frac{\gamma_{t h 1} \lambda_{S R} \lambda_{R U_{1}}}{\rho\left(a_{1}-2 \gamma_{t h 1} a_{2}\right)}}\right)
\end{aligned}
$$

\subsubsection{Outage probability of $U_{2}$}

Similarly, we first consider SINR in AF mode for $U_{2}$ as

$$
\gamma_{U_{2}}^{A F}=\frac{\rho a_{2}\left|h_{S R}\right|^{2}\left|h_{R U_{2}}\right|^{2}}{\left(1+\left|h_{R U_{2}}\right|\right)^{2}}
$$

Then, the outage probability can be determined at $U_{2}$ as

$$
\begin{aligned}
O P_{U_{2}}^{A F} & \simeq 1-2 e^{-\frac{\lambda_{S R} \gamma_{t h 2}}{\rho a_{2}}} \sqrt{\frac{\lambda_{S R} \lambda_{R U_{2}} \gamma_{t h 2}}{\rho a_{2}}} \\
& \times K_{1}\left(2 \sqrt{\frac{\lambda_{S R} \lambda_{R U_{2}} \gamma_{t h 2}}{\rho a_{2}}}\right)+e^{-\frac{\lambda_{S R} \gamma_{t h 2}}{\rho a_{2}}} \frac{4 \gamma_{t h 2} \lambda_{S R} \lambda_{R U_{2}}^{2}}{\rho a_{2}} \\
& \times\left(\frac{\gamma_{t h 2} \lambda_{S R}}{\rho a_{2} \lambda_{R U_{2}}}\right)^{1 / 4} K_{1 / 2}\left(2 \sqrt{\frac{\gamma_{t h 2} \lambda_{S R} \lambda_{R U_{2}}}{\rho a_{2}}}\right)
\end{aligned}
$$

By using (15) for (19), we formulate the outage probability as

$$
O P_{U_{1}}^{D F}=\operatorname{Pr}\left(\left|h_{S R}\right|^{2}<\frac{\gamma_{t h 1}\left(\left|h_{R U_{1}}\right|^{2}+1\right)}{\left(a_{1}-\gamma_{t h 1} a_{2}\right) \rho\left|h_{R U_{1}}\right|^{2}}\right)
$$



rewritten as

Based on probability density function (PDF) of $\left|h_{i}\right|^{2}$ is given as $f_{\left|h_{i}\right|^{2}}(x)=\lambda_{R U_{1}} e^{-\lambda_{R U_{1}} x}$, it can be

$$
\begin{aligned}
O P_{U_{1}}^{D F} & =\int_{0}^{\infty} f_{\left|h_{R U_{1}}\right|^{2}}(x) \int_{0}^{\frac{\gamma_{t h 1}(x+1)}{\left(a_{1}-\gamma_{t h 1} a_{2}\right) \rho x}} f_{\left|h_{S R}\right|^{2}}(y) d x d y \\
& =\int_{0}^{\infty}\left(1-e^{-\frac{\gamma_{t h 1}(x+1)}{\left(a_{1}-\gamma_{t h 1} a_{2}\right) \rho x}}\right) \lambda_{R U_{1}} e^{-\lambda_{R U_{1}} x} d x
\end{aligned}
$$

Thus, based on $[23,3.324 .1] O P_{U_{1}}^{D F}$ can be obtained as

$$
\begin{aligned}
O P_{U_{1}}^{D F} & =\int_{0}^{\infty}\left(1-e^{-\frac{\gamma_{t h 1}(x+1)}{\left(a_{1}-\gamma_{t h 1} a_{2}\right) \rho x}}\right) \lambda_{R U_{1}} e^{-\lambda_{R U_{1}} x} d x \\
& =1-2 e^{-\frac{\lambda_{S R} \gamma_{t h 1}}{\left(a_{1}-\gamma_{t h 1} a_{2}\right) \rho}} \sqrt{\frac{\gamma_{t h 1} \lambda_{S R} \lambda_{R U_{1}}}{\left(a_{1}-\gamma_{t h 1} a_{2}\right) \rho}} K_{1}\left(2 \sqrt{\frac{\gamma_{t h 1} \lambda_{R U_{1}} \lambda_{S R}}{\left(a_{1}-\gamma_{t h 1} a_{2}\right) \rho}}\right)
\end{aligned}
$$

It is end of the proof.

\section{SIMULATION RESULT}

In this section, we perform numerical simulations to exhibit performance metrics of EH-NOMA system. We set fixed power allocation factors as $a_{1}=0.9, a_{2}=0.1$. To form wireless channels, we set $\lambda_{S R}=\lambda_{R U_{2}}=1, \lambda_{R U_{1}}=2$.

The outage behavior of two users in DF and AF can be seen in Figure 2 and Figure 3. It can be confirmed that at high SNR, such outage performance can be improved significantly. Regardless architecture of AF and DF modes, similar trends of outage probability for two users can be reported. More importantly, theorical and Monte-Carlo simulation results are matched very tight which verify the correction of expression derived. Different power factors used in users are main reason of performance gap in Figure 2 and Figure 3.

In Figure 4, trends of outage probability are varied based on how percentage of power allocated to each user. When $a_{1}$ varies from 0.5 to 1 , outage probability of user $U_{1}$ improves clearly. Which situation is opposite with performance of user $U_{2}$.

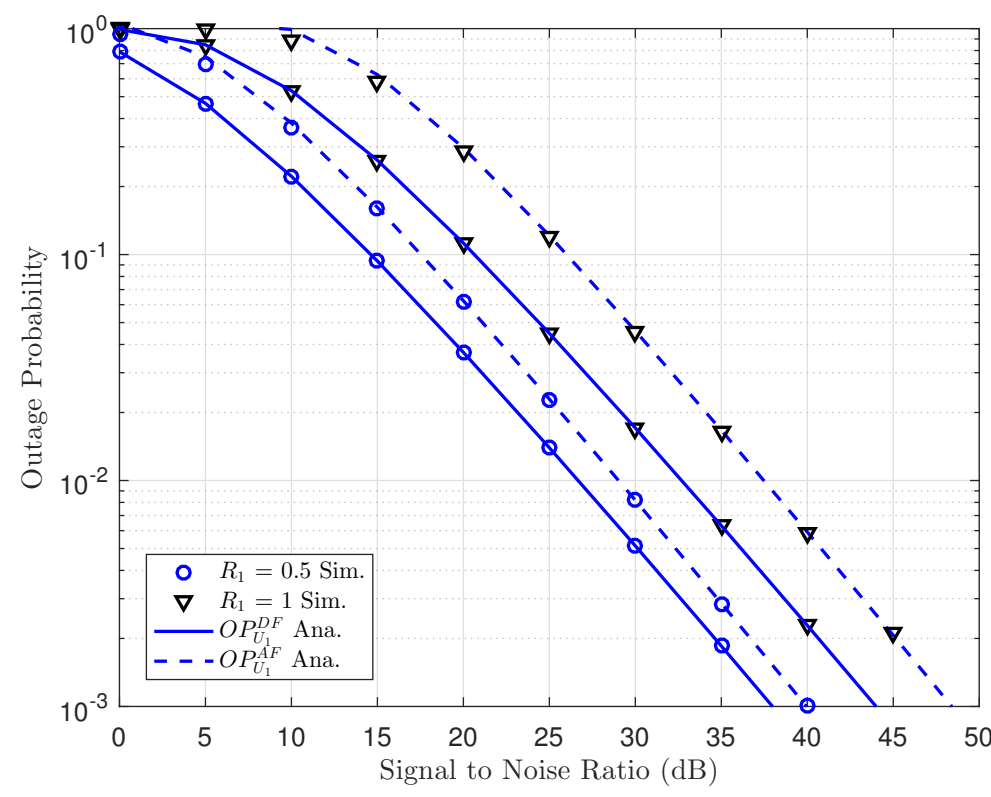

Figure 2. Outage performance of $U_{1}$ versus SNR with $R_{2}=0.5$ 


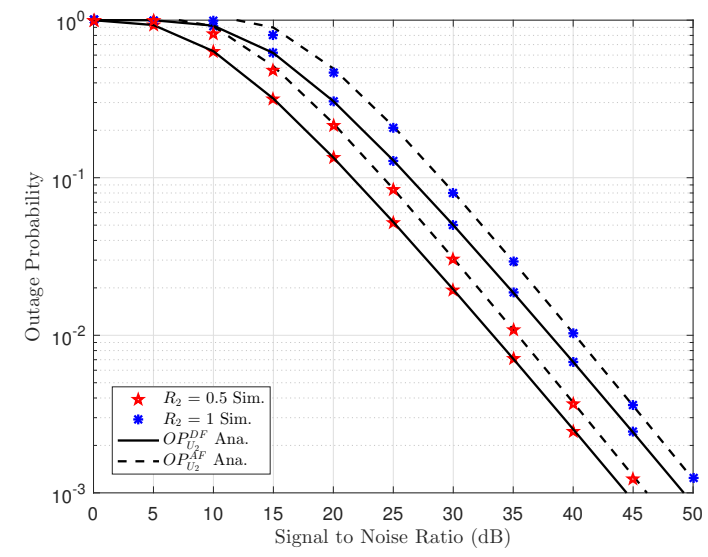

Figure 3. Outage performance of $U_{2}$ versus transmit SNR at BS with $R_{1}=0.5$

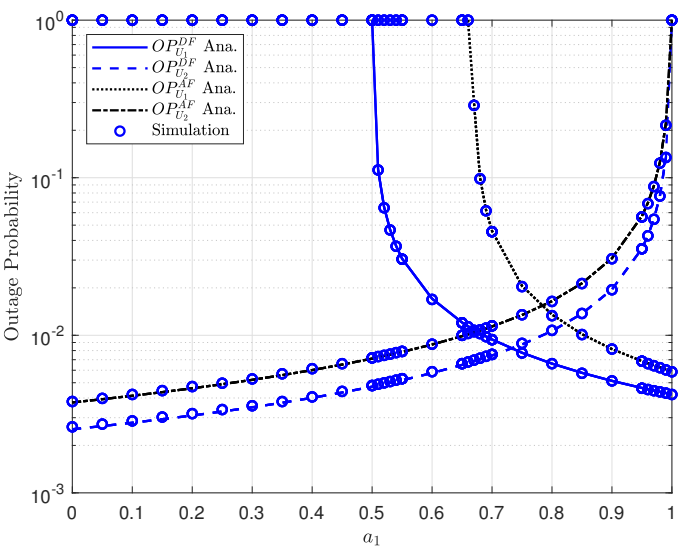

Figure 4. Outage performance of $U_{1}$ and $U_{2}$ versus $a_{1}$

\section{CONCLUSION}

This paper considers main parameters make influence on EH-NOMA system, in which NOMA users cooperate with relay by exploiting wireless powered capability. The detail performance analysis has been performed in terms of outage probability. The closed-form expressions related to such are derived, and it is shown that performance gap exists among two NOMA users for both AF and DF mode. The target rates and power allocation factors are main impacts on outage probability. By implement EH in such NOMA systems, we can prolong their operation and the constraint of outage performance is still acceptable.

\section{REFERENCES}

[1] Z. Ding, Z. Yang, P. Fan, and H. V. Poor, "On the performance of non-orthogonal multiple access in 5G systems with randomly deployed users," IEEE Signal Process. Lett., vol. 21, no.12 pp. 1501-1505, 2014.

[2] X. Li, J. Li, Y. Liu, Z. Ding and A. Nallanathan, "Residual Transceiver Hardware Impairments on Cooperative NOMA Networks," IEEE Transactions on Wireless Communications, vol. 19, no. 1, pp. 680-695, Jan. 2020.

[3] D.-T. Do, A.-T. Le and B.-M. Lee, "On Performance Analysis of Underlay Cognitive Radio-Aware Hybrid OMA/NOMA Networks with Imperfect CSI,” Electronics, vol. 8, no. 7, pp. 819, 2019.

[4] Dinh-Thuan Do, A. Le and B. M. Lee, "NOMA in Cooperative Underlay Cognitive Radio Networks Under Imperfect SIC," IEEE Access, vol. 8, pp. 86180-86195, 2020.

[5] J. Li, X. Li, Y. Liu, C. Zhang, L. Li and A. Nallanathan, "Joint Impact of Hardware Impairments and Imperfect Channel State Information on Multi-Relay Networks," in IEEE Access, vol. 7, pp. 72358-72375, 2019.

[6] L. Dai, B. Wang, M. Peng and S. Chen, "Hybrid Precoding-Based Millimeter-Wave Massive MIMO-NOMA With Simultaneous Wireless Information and Power Transfer," IEEE Journal on Selected Areas in Communications, vol. 37, no. 1, pp. 131-141, 2019.

[7] X. Li, J. Li and L. Li, ”Performance Analysis of Impaired SWIPT NOMA Relaying Networks Over Imperfect Weibull Channels," in IEEE Systems Journal, vol. 14, no. 1, pp. 669-672, March 2020.

[8] T.-L. Nguyen and Dinh-Thuan Do, "Power allocation schemes for wireless powered NOMA systems with imperfect CSI: An application in multiple antenna-based relay," International Journal of Communication Systems, vol. 31, no. 15, e3789, 2018.

[9] K. Tourki, H.-C. Yang, and M.-S. Alouini, "Accurate outage analysis of incremental decode-and-forward opportunistic relaying," IEEE Trans. Wireless Commun., vol. 10, no. 4, pp. 1021-1025, Apr. 2011.

[10] Dinh-Thuan Do, Chi-Bao Le and A.-T. Le, "Cooperative underlay cognitive radio assisted NOMA: secondary network improvement and outage performance," TELKOMNIKA (Telecommunication, Computing, Electronics and Control), vol. 17, no. 5, pp. 2147-2154, 2019.

[11] X. Li, M. Liu, C. Deng, P. T. Mathiopoulos, Z. Ding and Y. Liu, "Full-Duplex Cooperative NOMA Relaying Systems With I/Q Imbalance and Imperfect SIC," IEEE Wireless Communications Letters, vol. 9, no. 1, pp. 17-20, Jan. 2020.

[12] Dinh-Thuan Do and C.-B. Le, "Exploiting Outage Performance of Wireless Powered NOMA," TELKOMNIKA (Telecommunication, Computing, Electronics and Control), vol. 16, no. 5, pp. 1907-1917, 2018. 
[13] J. Wang, Q. Peng, Y. Huang, H.-M. Wang, and X. You, "Convexity of weighted sum rate maximization in NOMA systems," IEEE Commun.Lett., vol. 24, no. 9, pp. 1323-1326, Sep. 2017.

[14] X. Li, M. Huang, C. Zhang, D. Deng, K. M Rabie, Y. Ding, and J. Du, "Security and Reliability Performance Analysis of Cooperative Multi-Relay Systems With Nonlinear Energy Harvesters and Hardware Impairments," IEEE Access, vol. 7, pp. 102644-102661, 2019.

[15] D. Wang and S. Men, "Secure Energy Efficiency for NOMA Based Cognitive Radio Networks With Nonlinear Energy Harvesting," in IEEE Access, vol. 6, pp. 62707-62716, 2018.

[16] R. V. Bhat, M. Motani and T. J. Lim, "Hybrid NOMA for an Energy Harvesting MAC With Non-Ideal Batteries and Circuit Power," in IEEE Transactions on Wireless Communications, vol. 18, no. 8, pp. 3961-3973, Aug. 2019.

[17] X. Wang et al., "Energy Efficiency Optimization for NOMA-Based Cognitive Radio With Energy Harvesting," in IEEE Access, vol. 7, pp. 139172-139180, 2019.

[18] Z. Ni, Z. Chen, Q. Zhang and C. Zhou, "Analysis of RF Energy Harvesting in Uplink-NOMA IoT-Based Network," 2019 IEEE 90th Vehicular Technology Conference (VTC2019-Fall), Honolulu, HI, USA, pp. 1-5, 2019.

[19] A. Rauniyar, P. E. Engelstad and O. N. Østerb $\varnothing$, "Performance Analysis of RF Energy Harvesting and Information Transmission Based on NOMA With Interfering Signal for IoT Relay Systems," in IEEE Sensors Journal, vol. 19, no. 17, pp. 7668-7682, Sept, 2019.

[20] S. Mondal, S. Dhar Roy and S. Kundu, "Outage analysis for NOMA-based energy harvesting relay network with imperfect CSI and transmit antenna selection," in IET Communications, vol. 14, no. 14, pp. 2240-2249, 2020.

[21] S. Wang and T. Wu, "Stochastic Geometric Performance Analyses for the Cooperative NOMA With the Full-Duplex Energy Harvesting Relaying," in IEEE Transactions on Vehicular Technology, vol. 68, no. 5, pp. 4894-4905, May 2019.

[22] X. Li, M. Liu, C. Deng, , D. Zhang, X. Gao, K. M. Rabie, R. Kharel, "Joint Effects of Residual Hardware Impairments and Channel Estimation Errors on SWIPT Assisted Cooperative NOMA Networks," IEEE Access, pp.1-15, 2019.

[23] I. S. Gradshteyn and I. M. Ryzhik, "Table of Integrals, Series and Products," 6th ed. Academic Press, 2000.

[24] Alkheir, Ala Abu, and Mohamed Ibnkahla, "An accurate approximation of the exponential integral function using a sum of exponentials," IEEE Comm. Lett. vol. 17, no. 7, pp. 1364-1367, Jul. 2013.

[25] M. Ashraf, J. Jang, J. Han and K. G. Lee, "Capacity Maximizing Adaptive Power Splitting Protocol for Cooperative Energy Harvesting Communication Systems," IEEE Commun. Letters, vol. 22, no. 5, pp. 902-905, May 2018. 